\title{
ORBITS OF ALGEBRAIC NUMBERS WITH LOW HEIGHTS
}

\author{
GREGORY P. DRESDEN
}

\begin{abstract}
We prove that the two smallest values of $h(\alpha)+h\left(\frac{1}{1-\alpha}\right)+h\left(1-\frac{1}{\alpha}\right)$ are 0 and $0.4218 \ldots$, for $\alpha$ any algebraic integer.
\end{abstract}

\section{INTRODUCTION}

For $K$ an algebraic number field, let $K_{v}$ be the completion of $K$ at the place $v$ and let ||$_{v}$ be the absolute value associated with this completion $K_{v}$ (more precise definitions are given below). For $\alpha \in K$, we define the (logarithmic) Weil height, $h(\alpha)$, as follows:

$$
h(\alpha)=\sum_{v} \log \max \left(|\alpha|_{v}, 1\right) .
$$

In this paper, we will prove

Theorem 1. Let $\alpha$ be an algebraic number, $\alpha \neq 0,1$.

(i) For $\alpha$ a primitive sixth root of unity,

$$
h(\alpha)+h\left(\frac{1}{1-\alpha}\right)+h\left(1-\frac{1}{\alpha}\right)=0 .
$$

(ii) Otherwise,

$$
h(\alpha)+h\left(\frac{1}{1-\alpha}\right)+h\left(1-\frac{1}{\alpha}\right) \geq 0.4218 \ldots,
$$

with equality for $\alpha$ any root of the polynomial:

$$
\begin{aligned}
P_{1}(z) & =z^{6}-3 z^{5}+5 z^{4}-5 z^{3}+5 z^{2}-3 z+1 \\
& =\left(z^{2}-z+1\right)^{3}-\left(z^{2}-z\right)^{2} .
\end{aligned}
$$

The reader will note that this theorem is a specific case of the following general problem. We generalize the Weil height to $\mathbb{P}^{1}(\overline{\mathbb{Q}})$ in the obvious manner: for $\mathbf{x}=\left[\begin{array}{l}x_{1} \\ x_{2}\end{array}\right]$, we define

$$
h(\mathbf{x})=\sum_{v} \log \max \left(\left|x_{1}\right|_{v},\left|x_{2}\right|_{v}\right) .
$$

Received by the editor September 30, 1996.

1991 Mathematics Subject Classification. Primary 11R04, 11R06; Secondary 12D10.

I am very grateful for the assistance and guidance of my advisor, Dr. Vaaler.

(C)1998 American Mathematical Society 
Then, for $G$ a finite subgroup of $P G L_{2}(\overline{\mathbb{Q}})$, we extend the Weil height to orbits under the action of $G$, as follows:

$$
h_{G}(\mathbf{x})=\sum_{g \in G} h(g \mathbf{x}) .
$$

We now ask about the smallest values of $h_{G}(\mathbf{x})$ for $\mathbf{x} \in \mathbb{P}^{1}(\overline{\mathbb{Q}})$. We see that Theorem 1 answers this question for $G$ the cyclic group

$$
G=\left\{\left[\begin{array}{ll}
1 & 0 \\
0 & 1
\end{array}\right],\left[\begin{array}{cc}
0 & 1 \\
-1 & 1
\end{array}\right],\left[\begin{array}{cc}
1 & -1 \\
1 & 0
\end{array}\right]\right\}
$$

At the end of this paper we mention further work that is being done on other subgroups $G$ of $P G L_{2}(\overline{\mathbb{Q}})$.

The reader will also note that this theorem is related to a recent result by Zagier [7] in which he sharpens a result of Zhang [8] concerning a lower bound for $h(\alpha)+$ $h(1-\alpha)$.

Let us now proceed to a proof of Theorem 1.

\section{DEFINITIONS}

For $K_{v}$ the completion of the algebraic number field $K$ at the place $v$, we will need two absolute values, ||$_{v}$ (mentioned above) and \|\|$_{v}$. We define \|\|$_{v}$ to be the absolute value which, when restricted to $\mathbb{Q}$, is the usual Euclidean or $p$-adic absolute value, and we define ||$_{v}$ as follows

$$
||_{v}=\|\|_{v}^{d_{v} / d} \text {. }
$$

It follows that ||$_{v}$ satisfies the product formula on $K: \prod_{v}|\beta|_{v}=1$ for all non-zero $\beta \in K$. (Our normalizations of the absolute values are exactly as in [1] or [5].) Let us also agree that single-bar absolute values, || , without any subscript, will always refer to the usual Euclidean absolute value on $\mathbb{C}$. We will use the standard notation $\log ^{+}(z)$ to refer to $\max (0, \log (z))$. Finally, we will need to define the following function for our proof:

$$
E_{v}(z)=B \log \left\|\frac{\left(z^{2}-z+1\right)^{3}}{\left(z^{2}-z\right)^{2}}\right\|_{v}-\log ^{+}\|z\|_{v}-\log ^{+}\left\|\frac{1}{1-z}\right\|_{v}-\log ^{+}\left\|1-\frac{1}{z}\right\|_{v} .
$$

The constant $B$ will be specified later; it is a positive real number, between 0 and $1 / 2$. Notice that $E_{v}(z)$ is invariant under the transform $z \mapsto 1-\frac{1}{z}$; this means that

$$
E_{v}(z)=E_{v}\left(1-\frac{1}{z}\right)=E_{v}\left(\frac{1}{1-z}\right)
$$

In our proof of Theorem 1, we first establish some local estimates, and we then use these to establish a global result that will prove the theorem.

\section{LOCAL ESTIMATES}

Lemma 1. Let $z$ be an algebraic number, $z \neq 0,1$, or a primitive sixth root of unity.

(i) $E_{v}(z) \leq 0$ for $v$ finite, with equality for $z$ any root of $P_{1}$.

(ii) $E_{v}(z) \leq-0.4218 \ldots$ for $v$ infinite, with equality for $z$ any root of $P_{1}$. 
Proof of Lemma 1. The two parts of this lemma will require entirely different techniques to prove. In (i), for $v$ finite, we will rely on the triangle inequality property of \|\|$_{v}$, and in (ii), we will differentiate $E_{v}(z)$ and solve for $z$. (In both parts, we assume that $z$ is neither 0 nor 1.)

Proof of part (i): $v$ finite. Recall the ultrametric triangle inequality: $\|a+b\|_{v} \leq$ $\max \left(\|a\|_{v},\|b\|_{v}\right)$, and if $\|a\|_{v} \neq\|b\|_{v}$, then $\|a+b\|_{v}=\max \left(\|a\|_{v},\|b\|_{v}\right)$.

For $\Phi_{6}(z)=z^{2}-z+1$, we have the following interesting identity:

$$
\Phi_{6}(z) \Phi_{6}\left(\frac{1}{1-z}\right) \Phi_{6}\left(1-\frac{1}{z}\right)=\frac{\left(z^{2}-z+1\right)^{3}}{\left(z^{2}-z\right)^{2}} .
$$

For finite $v$, then $\left\|\Phi_{6}(z)\right\|_{v} \leq \max \left(1,\left\|z^{2}\right\|_{v}\right)$, and so $\log \left\|\Phi_{6}(z)\right\|_{v} \leq 2 \log ^{+}\|z\|_{v}$. Thus, if we apply \|\|$_{v}$ to both sides of (6) and then take the logarithm, we conclude:

$$
2 \log ^{+}\|z\|_{v}+2 \log ^{+}\left\|\frac{1}{1-z}\right\|_{v}+2 \log ^{+}\left\|1-\frac{1}{z}\right\|_{v} \geq \log \left\|\frac{\left(z^{2}-z+1\right)^{3}}{\left(z^{2}-z\right)^{2}}\right\|_{v} .
$$

Since the constant $B$ in equation (4) is less than $1 / 2$, this implies that $E_{v}(z) \leq 0$.

It remains to show that equality is achieved for $z$ a root of the polynomial $P_{1}$. Let $z_{1}$ be such a root. It is easy to show that $1-\frac{1}{z_{1}}$ and $\frac{1}{1-z_{1}}$ are also roots of $P_{1}$, and since $P_{1}$ is a monic polynomial with integer coefficients and a constant coefficient of 1 , then all of its roots are algebraic units. This implies that all three of the $\log ^{+}$terms in $E_{v}\left(z_{1}\right)$ are zero; the first term is clearly zero as well, and thus $E_{v}\left(z_{1}\right)=0$.

Proof of part (ii): $v$ infinite. We need to define a new constant, $D$, in terms of $B$ :

$$
D=\frac{1}{2}[(1+2 B) \log (1+2 B)-(6 B) \log (6 B)-(1-4 B) \log (1-4 B)] .
$$

We now describe the method used to determine the value of $B$. This constant $B$ is chosen so as to maximize the value of $D$; by differentiating (8) and solving, we find that $B$ should be the single real root of the polynomial $184 x^{3}+6 x-1$. That is, $B=0.1172 \ldots$, and subsequently, $D=0.4218 \ldots$ (Notice that $-D$ is the number appearing in the statement of Lemma 1, part (ii).)

Let us now show that $E_{v}(z) \leq-D$ for all $z \in \mathbb{C}$. Recall that for $v$ infinite, then \|\|$_{v}=||$, the regular Euclidean absolute value on $\mathbb{C}$.

Since $E_{v}(z)$ goes to $-\infty$ for $z$ near $0,1, \infty$, and the primitive sixth roots of unity, and since $E_{v}(z)$ is harmonic off the three curves $|z|=1,\left|1-\frac{1}{z}\right|=1$, and $\left|\frac{1}{1-z}\right|=1$, then (by the maximum principle) $E_{v}(z)$ achieves its maximum only on these three curves. By the invariance expressed in equation (5), we need only check one of these curves. We consider the straight line $\left|1-\frac{1}{z}\right|=1$, which is easily parametrized by $z=1 / 2+i y$. Since $E_{v}(z)=E_{v}(\bar{z})$, we need only consider $y \geq 0$. We substitute our parametrization into $E_{v}(z)$ and derive the following formula:

$$
E_{v}(1 / 2+i y)= \begin{cases}3 B \log \left(\frac{3}{4}-y^{2}\right)+\left(\frac{1}{2}-2 B\right) \log \left(\frac{1}{4}+y^{2}\right) & \text { for } y \in\left(0, \frac{\sqrt{3}}{2}\right), \\ 3 B \log \left(y^{2}-\frac{3}{4}\right)-\left(\frac{1}{2}+2 B\right) \log \left(\frac{1}{4}+y^{2}\right) & \text { for } y \in\left(\frac{\sqrt{3}}{2}, \infty\right)\end{cases}
$$

If we let $S=y^{2}+1 / 4$, then (9) becomes

$$
E_{v}(z)= \begin{cases}3 B \log (1-S)+\left(\frac{1}{2}-2 B\right) \log (S) & \text { for } S \in\left(\frac{1}{4}, 1\right) \\ 3 B \log (S-1)-\left(\frac{1}{2}+2 B\right) \log (S) & \text { for } S \in(1, \infty)\end{cases}
$$


We now find the maximum of $E_{v}(z)$ by differentiating (10) with respect to $S$, setting the result equal to zero, and solving for $S$. We find that $E_{v}(z)$ has two maxima, at

$$
S_{1}=\frac{1-4 B}{1+2 B}, \quad \text { and } \quad S_{2}=\frac{1+4 B}{1-2 B} .
$$

Using our value of $B$ we can compute that $S_{1} \in(1 / 4,1)$ and $S_{2} \in(1, \infty)$. That both points are (local) maxima for $E_{v}(z)$ can easily be verified by the second derivative test.

We substitute $S_{1}$ and $S_{2}$ into $E_{v}$ and find the following:

$$
\begin{aligned}
E_{v}\left(S_{1}\right) & =\frac{1}{2}[(6 B) \log (6 B)+(1-4 B) \log (1-4 B)-(1+2 B) \log (1+2 B)] \\
& =-D
\end{aligned}
$$

and,

$$
\begin{aligned}
E_{v}\left(S_{2}\right) & =\frac{1}{2}[(6 B) \log (6 B)-(1+4 B) \log (1+4 B)+(1-2 B) \log (1-2 B)] \\
& <-D
\end{aligned}
$$

Thus, the maximum value for $E_{v}(z)$ is $-D$. This value is attained at $S_{1}=$ $(1-4 B)(1+2 B)^{-1}$, and since $B$ is a root of $184 x^{3}+6 x-1$, we find that $S_{1}$ satisfies

$$
S_{1}^{3}-2 S_{1}^{2}+3 S_{1}-1=0
$$

If we recall that $S_{1}=y^{2}+1 / 4$, and $z=1 / 2+i y$, then we see that $S_{1}$ represents the algebraic number $z$ that is a root of the polynomial

$$
z^{6}-3 z^{5}+5 z^{4}-5 z^{3}+5 z^{2}-3 z+1=0 .
$$

This is exactly the polynomial $P_{1}(z)$ from equation (2). We have thus shown that $E_{v}(z) \leq-D$, and $E_{v}(z)=-D$ for $z$ a root of $P_{1}(z)$. Of course, $P_{1}$ has five other roots; these are also maximums for $E_{v}(z)$ and reflect the invariance of $E_{v}$ from equation (5) and the fact that $E_{v}(z)=E_{v}(\bar{z})$.

\section{Global ESTIMATES}

We will now combine our local estimates to prove Theorem 1.

We first need to introduce a new constant, $n_{v}$, defined as $n_{v}=0$ for $v$ finite, and $n_{v}=d_{v} / d$ for $v$ infinite. We now combine (i) and (ii) of Lemma 1 into a single statement:

$$
d_{v} / d E_{v}(z) \leq-n_{v} D
$$

Proof of Theorem 1. In equation (13), we multiply each logarithm in $E_{v}(z)$ by the $d_{v} / d$ term, and use the relation expressed in equation (3), to produce:

$$
B \log \left|\frac{\left(z^{2}-z+1\right)^{3}}{\left(z^{2}-z\right)^{2}}\right|_{v}-\log ^{+}|z|_{v}-\log ^{+}\left|\frac{1}{1-z}\right|_{v}-\log ^{+}\left|1-\frac{1}{z}\right|_{v} \leq-n_{v} D .
$$

We now make use of the identities

$$
\sum_{v} n_{v}=1, \quad \sum_{v} \log |\beta|_{v}=0, \quad \sum_{v} \log ^{+}|\beta|_{v}=h(\beta) .
$$


(These last two formulas hold for all non-zero algebraic numbers, $\beta$ ). Then, for $z$ not zero, 1 , or a primitive sixth root of unity, we can sum (14) over all places $v$ and apply (15) to get

$$
-h(z)-h\left(\frac{1}{1-z}\right)-h\left(1-\frac{1}{z}\right) \leq-D .
$$

This implies

$$
h(z)+h\left(\frac{1}{1-z}\right)+h\left(1-\frac{1}{z}\right) \geq D=0.4218 \ldots,
$$

and since equality holds in (13) for $z$ any root of $P_{1}$, the same can be said of (16). This establishes part (ii) of Theorem 1; as for part (i), it follows easily from the fact that the minimal polynomial of the sixth roots of unity is $z^{2}-z+1$.

\section{ApPLiCATIONS AND GENERALIZATIONS}

It is interesting to note that the Weil height $h$ is related to the Mahler measure of a polynomial (as seen in [2] or [3]). Recall that for a polynomial $f(x)=a_{0}+$ $a_{1} x+a_{2} x^{2}+\cdots+a_{n} x^{n}$, with zeroes at $\alpha_{1}, \ldots, \alpha_{n}$, we define the Mahler measure $M(f)$ to be

$$
M(f)=\left|a_{n}\right| \prod_{i=1}^{n} \max \left(\left|\alpha_{i}\right|, 1\right) .
$$

D. Lehmer [4] asked if there exists a non-trivial lower bound to $M(f)$ for $f$ not cyclotomic (it is conjectured that this lower bound is $1.17628 \ldots$. ). The exact relationship between the Weil height and the Mahler measure is as follows [7]. For $\alpha_{i}$ a root of the polynomial $f(x)$, then

$$
h\left(\alpha_{i}\right)=\frac{1}{\operatorname{deg} f} \log M(f) .
$$

Given this relation, one can establish an immediate corollary to Theorem 1 . Let $G$ be the cyclic group of order three, generated by $z \mapsto 1-1 / z$. Let $f(x) \in \mathbb{Z}[x]$ be a polynomial of degree $n$ such that $G$ is a subgroup of its Galois group. Then,

$$
M(f) \geq e^{n k}
$$

where $k$ is $\frac{1}{3}(0.4218 \ldots)$. One can compare this to the result of Dobrowolski [3], later improved by Rausch $[6]$, that for $g(x) \in \mathbb{Z}[x]$ any non-cyclotomic polynomial of degree $n$, then

$$
M(g) \geq 1+b\left(\frac{\log \log n}{\log n}\right)^{3}
$$

for $b$ a small positive constant.

Let us now return to the generalization of Theorem 1, mentioned earlier in this paper. It is certainly possible to extend this result to other subgroups of $P G L_{2}(\overline{\mathbb{Q}})$; consider the subgroup $K$ defined as

$$
K=\left\{\left[\begin{array}{ll}
1 & 0 \\
0 & 1
\end{array}\right],\left[\begin{array}{cc}
1 & 1 \\
-1 & 1
\end{array}\right],\left[\begin{array}{cc}
0 & -1 \\
1 & 0
\end{array}\right],\left[\begin{array}{cc}
1 & -1 \\
1 & 1
\end{array}\right]\right\}
$$

Then, in a proof similar to the proof of Theorem 1 , we can show that $h_{K}(\mathbf{x})=0$ for $x=\left[\begin{array}{l}i \\ 1\end{array}\right],\left[\begin{array}{c}-i \\ 1\end{array}\right]$, or any element in the orbit of $\left[\begin{array}{l}0 \\ 1\end{array}\right]$ under $K$; and that otherwise 
$h_{K}(\mathbf{x}) \geq 0.732858 \ldots$, with equality at $\mathbf{x}$ a root of the homogeneous polynomial $x_{1}^{8}+5 x_{1}^{6} x_{2}^{2}+4 x_{1}^{4} x_{2}^{4}+5 x_{1}^{2} x_{2}^{6}+x_{2}^{8},=\left(x_{1}^{2}+x_{2}^{2}\right)^{4}+\left(\left(x_{1} x_{2}\right)\left(x_{1}+x_{2}\right)\left(x_{1}-x_{2}\right)\right)^{2}$.

An interesting problem would be to specify for which other subgroups $G$ of $P G L_{2}(\overline{\mathbb{Q}})$ one can find a similar statement.

It would also be interesting to determine if one can find other low values in the spectrum of $h_{G}$ for a given group $G$, along with the exact algebraic numbers which achieve those values. For our original group $G$ of order 3, after the first non-zero value of $0.4218 \ldots$, the author conjectures that the next two values in the spectrum of $h_{G}$ are $0.43359381 \ldots$ and $0.43798825 \ldots$

I wish to thank Dr. Vaaler for his many helpful comments, and also for suggesting the identity in formula (6).

\section{REFERENCES}

1. E. Bombieri, A van der Poorten, and J. Vaaler, Effective measures of irrationality for cubic extensions of number fields, Ann. Scuola Norm. Sup. Pisa Cl. Sci. (4) 23 (1996), 211-248. CMP 97:08

2. D. Boyd, Speculations concerning the range of Mahler's Measure, Canad. Math. Bull. 24 (4) (1981), 453-469. MR 83h:12002

3. E. Dobrowolski, On a question of Lehmer and the number of irreducible factors of a polynomial, Acta Arithmetica 34 (1979), 391-401. MR 80i:10040

4. D. H. Lehmer, Factorization of certain cyclotomic funcions, Ann. of Math. 34 (1933), 461479 .

5. C. Pinner and J. Vaaler, The number of irreducible factors of a polynomial, I, Trans. Amer. Math. Soc. 339 (1993), 809-834. MR 93m:11108

6. U. Rausch, On a theorem of Dobrowolski about the product of conjugate numbers, Colloquium Mathematicum 50 (1985), 137-142. MR 87i:11144

7. D. Zagier, Algebraic numbers close to both 0 and 1, Mathematics of Computation 61 (203) (1993), 485-491. MR 94c:11104

8. S. Zhang, Positive line bundles on arithmetic surfaces, Ann. of Math. (2) 136 (3) (1992), 569-587. MR 93j:14024

Department of Mathematics, Washington \& Lee University, Lexington, Virginia 24450

E-mail address: dresdeng@wlu.edu 